\title{
EXPONENTS AND DIAMETERS OF STRONG PRODUCTS OF DIGRAPHS*
}

\author{
BYEONG MOON $\mathrm{KIM}^{\dagger}$, BYUNG CHUL SONG ${ }^{\dagger}$, AND WOONJAE HWANG ${ }^{\ddagger}$
}

Abstract. The exponent of the strong product of a digraph of order $m$ and a digraph of order $n$ is shown to be bounded above by $m+n-2$, with equality for $Z_{m} \otimes Z_{n}$. The exponent and diameter of the strong product of a graph and a digraph are also investigated.

Key words. Strong product of digraphs, Exponent, Diameter.

AMS subject classifications. 15A48, 05C50, 05C76.

1. Introduction. Let $D=(V, A)$ be a digraph on $n$ vertices. Throughout this paper, we assume that $D$ has no loops and no multiple arcs. A walk from $u$ to $v$ in $D$ is a sequence $u=u_{0}, u_{1}, \ldots, u_{k}=v$ of vertices such that there is an arc from $u_{i}$ to $u_{i+1}$ in $D$ for each $i$. We denote the walk by $u \rightarrow u_{1} \rightarrow u_{2} \rightarrow \cdots \rightarrow u_{k-1} \rightarrow v$ and its length is $k$. We use the notation $u \stackrel{k}{\longrightarrow} v$ when there exits a walk in $D$ of length $k$ from $u$ to $v$. The digraph is primitive if there is a $k$ such that $u \stackrel{k}{\longrightarrow} v$ for each pair of vertices $u$ and $v$. Conventionally $u \stackrel{0}{\longrightarrow} u$ is permitted. We say that the smallest such value of $k$ is the exponent of $D$, which is denoted by $\exp (D)$. Wielandt [9] found that the maximum exponent of a primitive digraph on $n$ vertices is $W_{n}=n^{2}-2 n+2$. See [1] for more details. Suppose that two digraphs $D=\left(V_{D}, A_{D}\right)$ and $E=\left(V_{E}, A_{E}\right)$ are given. Let $V=V_{D} \times V_{E}$. We define

$$
\begin{gathered}
A_{1}=\left\{\left(\left(u_{1}, u_{2}\right),\left(v_{1}, v_{2}\right)\right) \in V \times V \mid\left(\left(u_{1}, v_{1}\right) \in A_{D} \text { and } u_{2}=v_{2}\right)\right. \\
\text { or } \left.\left(\left(u_{2}, v_{2}\right) \in A_{E} \text { and } u_{1}=v_{1}\right)\right\}
\end{gathered}
$$

and

$$
A_{2}=\left\{\left(\left(u_{1}, u_{2}\right),\left(v_{1}, v_{2}\right)\right) \in V \times V \mid\left(u_{1}, v_{1}\right) \in A_{D} \text { and }\left(u_{2}, v_{2}\right) \in A_{E}\right\} .
$$

The strong product $D \otimes E$ of $D$ and $E$ is the digraph $\left(V, A_{1} \cup A_{2}\right)$. The Cartesian product $D \times E$ and the direct product $D \otimes E$ of $D$ and $E$ are defined by $\left(V, A_{1}\right)$

\footnotetext{
*Received by the editors on July 24, 2010. Accepted for publication on October 24, 2011. Handling Editor: Bryan L. Shader.

${ }^{\dagger}$ Department of Mathematics, Gangneung-Wonju National University, Gangneung 210-702, Korea (kbm@gwnu.ac.kr, bcsong@gwnu.ac.kr).

$\ddagger$ Department of Information and Mathematics, Korea University, Jochiwon 339-700, Korea (woonjae@korea.ac.kr).
} 
and $\left(V, A_{2}\right)$ respectively. The strong product of graphs is used to define the concept of Shannon capacity which plays an important role in the information theory [8]. Definitions and related results on the product of graphs are provided in [2]. In 1979, Lamprey and Barnes [6] showed that $\exp (D \times E) \leq(n+m)^{2}-4(n+m)+5$ for digraphs $D$ and $E$ on $n$ and $m$ vertices, respectively. They also showed $\exp (D \otimes E)=$ $\max \{\exp (D), \exp (E)\}$ for primitive digraphs $D$ and $E$. In 1987, Kwasnik [5] studied the exponent of other types of products such as the disjunction and lexicographic products of graphs. Recently, it has been proved in [3] that if $D$ and $E$ are digraphs on $m$ and $n$ vertices, respectively, and $D \times E$ is primitive, then $\exp (D \times E) \leq m n-1$. In [3] it was also showed that $\exp (G \times D)=\exp (G)+\operatorname{diam}(D)$ for a primitive graph $G$ and a strongly connected bipartite digraph $D$, and they computed the exponent of the Cartesian product of two cycles [4]. In this paper, we show

$$
\exp (D \otimes E) \leq n+m-2
$$

for strongly connected digraphs $D$ and $E$ on $n$ and $m$ vertices, respectively. Let $Z_{n}$ and $Z_{m}$ be the directed cycles of order $n$ and $m$ respectively. We also prove that

$$
\exp \left(Z_{n} \otimes Z_{m}\right)=n+m-2 .
$$

As a consequence, the bound in (1.1) is tight. A graph $G$ is considered as a digraph by treating the edges of $G$ as bidirectional. In particular, a cycle $C_{n}$ of length $n$ is considered as a digraph in the same manner. For a connected graph $G$ and a strongly connected digraph $E$, we show $\exp (G \otimes E)$ is $\operatorname{diam}(G \otimes E)$ or $\operatorname{diam}(G \otimes E)+1$, and we find the condition under which the latter case holds. As a consequence, we compute $\exp \left(C_{n} \otimes Z_{m}\right)$.

\section{Upper bound on the exponent of strong products of two digraphs.}

Lemma 1. Let $D$ and $E$ be digraphs, $u, v \in V_{D}$ and $z, w \in V_{E}$. If $u \stackrel{t}{\longrightarrow} v$ in $D$ and $z \stackrel{s}{\longrightarrow} w$ in $E$, then $(u, z) \stackrel{\alpha}{\longrightarrow}(v, w)$ in $D \otimes E$ for all $\alpha$ with $\max \{t, s\} \leq \alpha \leq t+s$.

Proof. We may assume that $t \leq s$. Let $u \rightarrow u_{0} \rightarrow u_{1} \rightarrow \cdots \rightarrow u_{t}=v$ in $D$ and $z=z_{0} \rightarrow z_{1} \rightarrow \cdots \rightarrow z_{s}=w$ in $E$. If $i=\alpha-s$ for $0 \leq i \leq t$, then $(u, z)=\left(u_{0}, z_{0}\right) \rightarrow$ $\left(u_{1}, z_{1}\right) \rightarrow \cdots \rightarrow\left(u_{t-i}, z_{t-i}\right) \rightarrow\left(u_{t-i}, z_{t-i+1}\right) \rightarrow \cdots \rightarrow\left(u_{t-i}, z_{s}\right) \rightarrow\left(u_{t-i+1}, z_{s}\right) \rightarrow$ $\cdots \rightarrow\left(u_{t}, z_{s}\right)=(v, w)$ is a walk of length $t-i+s-(t-i)+t-(t-i)=s+i=\alpha$ in $D \otimes E$. $\square$

LEMmA 2. Let $D$ and $E$ be strongly connected digraphs, $u, v \in V_{D}$ and $z, w \in V_{E}$. If there are a cycle $C$ passing through $v$ of length $k$ in $D, u \stackrel{t}{\longrightarrow} v^{\prime}$ in $D$ for some vertex $v^{\prime}$ of $C$, and $z \stackrel{s}{\longrightarrow} w$ in $E$ for some $s \geq k-1$, then, for all $\alpha$ with $\max \{t+k, s\} \leq \alpha$, $(u, z) \stackrel{\alpha}{\longrightarrow}(v, w)$ in $D \otimes E$.

Proof. Since $v$ and $v^{\prime}$ are vertices of $C, v^{\prime} \stackrel{l}{\longrightarrow} v$ in $D$ for some $l$ with $0 \leq l \leq k-1$. So $t+l \leq t+k$. Since $\alpha-t-l \geq \alpha-t-k \geq 0$, there is a $q \geq 0$ such that 
$k q \leq \alpha-t-l \leq k q+k-1$. Since $u \stackrel{t}{\longrightarrow} v^{\prime} \stackrel{l}{\longrightarrow} v \stackrel{k q}{\longrightarrow} v, u \stackrel{k q+t+l}{\longrightarrow} v$ in $D$. Since $z \stackrel{s}{\longrightarrow} w$ in $E$ and $\max \{k q+t+l, s\} \leq \alpha \leq k q+t+l+k-1 \leq k q+t+l+s$, by Lemma $1,(u, z) \stackrel{\alpha}{\longrightarrow}(v, w)$ in $D \otimes E$.

THEOREM 1. Let $D$ and $E$ be strongly connected digraphs on $n$ and $m$ vertices $(n, m \geq 2)$, respectively. Then $D \otimes E$ is primitive and

$$
\exp (D \otimes E) \leq n+m-2
$$

Proof. It suffices to show that for each pair of vertices $(u, z),(v, w) \in D \otimes E$ and for each $\alpha \geq n+m-2$, we have $(u, z) \stackrel{\alpha}{\longrightarrow}(v, w)$ in $D \otimes E$. Let $k$ be the minimum length of the cycles in $D$ passing through $v$, and $C$ be one such cycle. Let $t$ be the distance from $u$ to $C$ in $D$. Then $k+t \leq n$.

Let $l$ be the minimum length of the cycle in $E$ passing through $w$ and $s$ be the distance from $z$ to $w$. Then $l, s \leq m$. If $s \geq k-1$, then, by $\alpha \geq n \geq t+k$ and $\alpha \geq m \geq s$, Lemma 2 implies that $(u, z) \stackrel{\alpha}{\longrightarrow}(v, w)$. If $s<k-1$, then there is a $q \geq 0$ such that $l q<k-s-1 \leq l(q+1)$. Then $l(q+1)+s=l+l q+s \leq l+k-2 \leq$ $m+k-2 \leq n+m-2 \leq \alpha$. Since $z \stackrel{s}{\longrightarrow} w \stackrel{l(q+1)}{\longrightarrow} w, z \stackrel{l(q+1)+s}{\longrightarrow} w$. By Lemma 2, $(u, z) \stackrel{\alpha}{\longrightarrow}(v, w)$.

TheOREM 2. For $n, m \geq 2$,

$$
\exp \left(Z_{n} \otimes Z_{m}\right)=n+m-2
$$

Proof. Let $Z_{n}$ be a directed cycle $v_{0} \rightarrow v_{1} \rightarrow \cdots \rightarrow v_{n-1} \rightarrow v_{0}$ and $Z_{m}$ be a directed cycle $w_{0} \rightarrow w_{1} \rightarrow \cdots \rightarrow w_{m-1} \rightarrow w_{0}$. We may assume $n \leq m$. Let $r$ be the residue of $m-2$ modulo $n$. Suppose $\left(v_{0}, w_{0}\right) \stackrel{n+m-3}{\longrightarrow}\left(v_{r}, w_{n-2}\right)$. Let $\left(v_{0}, w_{0}\right)=$ $\left(x_{0}, y_{0}\right) \rightarrow\left(x_{1}, y_{1}\right) \rightarrow \cdots \rightarrow\left(x_{n+m-3}, y_{n+m-3}\right)=\left(v_{r}, w_{n-2}\right)$ be a path in $Z_{n} \otimes Z_{m}$ from $\left(v_{0}, w_{0}\right)$ to $\left(v_{r}, w_{n-2}\right)$. Then there are $i_{0}<i_{1}<\cdots<i_{s}$ and $j_{0}<j_{1}<\cdots<j_{t}$ such that $i_{0}=j_{0}=0$, for all $p, q$ with $0 \leq p \leq s-1$ and $0 \leq q \leq t-1, x_{i_{p}}=$ $x_{i_{p}+1}=\cdots=x_{i_{p+1}-1} \neq x_{i_{p+1}}, y_{j_{q}}=y_{j_{q}+1}=\cdots=y_{j_{q+1}-1} \neq y_{j_{q+1}}$ and $x_{i_{s}}=x_{i_{s}+1}=$ $\cdots=x_{n+m-3}, y_{j_{t}}=y_{j_{t}+1}=\cdots=y_{n+m-3}$. Then for all $i=0,1, \ldots, n+m-3$, $\left(x_{i}, y_{i}\right)=\left(x_{i_{p}}, y_{j_{q}}\right)$ for some $p$ and $q$. If $0 \leq i \leq n+m-4$ and $\left(x_{i}, y_{i}\right)=\left(x_{i_{p}}, y_{j_{q}}\right)$, since $\left(x_{i+1}, y_{i+1}\right) \neq\left(x_{i}, y_{i}\right), x_{i+1}=x_{i_{p+1}}$ or $y_{i+1}=y_{j_{q+1}}$. So if $\left(x_{i}, y_{i}\right)=\left(x_{i_{p}}, y_{j_{q}}\right)$, we can show $i \leq p+q$, by induction. If $x_{i_{p}}=v_{l}$ and $l \neq n-1$, since $\left(x_{i_{p}}, x_{i_{p+1}}\right) \in A_{Z_{n}}$, $x_{i_{p+1}}=v_{l+1}$. If $x_{i_{p}}=v_{n-1}$, since $x_{i_{p}}=v_{n-1} \rightarrow x_{i_{p+1}}, x_{i_{p+1}}=v_{0}$. Since $x_{i_{0}}=v_{0}$, we can show by induction that if $x_{i_{p}}=v_{l}, p \equiv l(\bmod n)$. Similarly, we can show that if $y_{j_{q}}=w_{k}, q \equiv k(\bmod m)$. Since $y_{j_{t}}=w_{n-2}, t \equiv n-2(\bmod m)$. Since $t \leq n+m-3$ and $n \leq m, t=n-2$. Since $x_{i_{s}}=v_{r}, s \equiv r(\bmod n)$. So $s \equiv r \equiv m-2(\bmod n)$. 
Since $s \leq n+m-3, s \leq m-2$. So $n+m-3 \leq s+t \leq(m-2)+(n-2)=n+m-4$. This is a contradiction. So $\left(v_{0}, w_{0}\right) \stackrel{n+m-3}{\rightarrow}\left(v_{r}, w_{n-2}\right)$. Thus, using Theorem 1 , $\exp \left(Z_{n} \otimes Z_{m}\right)=n+m-2$.

3. Exponents and diameters of strong products of digraphs. For any $u, v \in V_{D}$, the distance $\operatorname{dist}(u, v)$ from $u$ to $v$ is the smallest $k$ such that there is a walk from $u$ to $v$ of length $k$. The diameter $\operatorname{diam}(D)$ of the strongly connected digraph $D$ is the maximum of $\operatorname{dist}(u, v)$ for all $u, v \in V_{D}$.

Proposition 1. If $D$ and $E$ are strongly connected digraphs, then

$$
\operatorname{diam}(D \otimes E)=\max \{\operatorname{diam}(\mathrm{D}), \operatorname{diam}(\mathrm{E})\}
$$

Proof. If $u, v \in V_{D}$ and $z, w \in V_{E}$, then by Lemma 1, we have

$$
\operatorname{dist}((u, z),(v, w)) \leq \max \{\operatorname{dist}(u, v), \operatorname{dist}(z, w))\} .
$$

Thus, $\operatorname{diam}(D \otimes E) \leq \max \{\operatorname{diam}(D), \operatorname{diam}(E))\}$.

Conversely, if $u, v \in V_{D}, z, w \in V_{E}$, and $\operatorname{dist}((u, z),(v, w))=\alpha$, then $(u, z)=$ $\left(u_{0}, z_{0}\right) \rightarrow\left(u_{1}, z_{1}\right) \rightarrow \cdots \rightarrow\left(u_{\alpha}, z_{\alpha}\right)=(v, w)$ for some $\left(u_{i}, z_{i}\right) \in V_{D \otimes E}$ where $i=1,2, \ldots, \alpha$. Thus, there are $0=i_{0}<i_{1}<i_{2}<\cdots<i_{s} \leq \alpha$ such that $u_{i_{p}}=u_{i_{p}+1}=\cdots=u_{i_{p+1}-1} \neq u_{i p+1}$ for all $p=0,1, \ldots, s-1$. Since $u=$ $u_{i_{0}} \rightarrow u_{i_{1}} \rightarrow \cdots \rightarrow u_{i_{s}}=u_{\alpha}=v$, $\operatorname{dist}(u, v) \leq s \leq \alpha=\operatorname{dist}((u, z),(v, w))$. So $\operatorname{diam}(D) \leq \operatorname{diam}(D \otimes E)$. Similarly, $\operatorname{diam}(E) \leq \operatorname{diam}(D \otimes E)$. Thus, $\operatorname{diam}(D \otimes E)=$ $\max \{\operatorname{diam}(D), \operatorname{diam}(E))\}$.

Lemma 3. Let $G$ be a connected graph and $D$ be a strongly connected digraph. If $(u, z),(v, w) \in V_{G \otimes D},((u, z),(v, w)) \in A_{G \otimes D}$ and $z \neq w$, then $(u, z) \stackrel{k}{\longrightarrow}(v, w)$ in $G \otimes D$ for all $k \geq 1$.

Proof. Since $z \neq w,(z, w) \in A_{D}$. Since $G$ is connected, there is $x \in V_{G}$ such that $\{u, x\} \in E_{G}$. Since $(u, z) \rightarrow(x, z) \rightarrow(u, z),(u, z) \stackrel{2 t}{\longrightarrow}(u, z)$ for all $t \geq 0$. If $u=v$, since $(u, z) \stackrel{2 t}{\longrightarrow}(u, z) \stackrel{1}{\longrightarrow}(u, w)$ and $(u, z) \stackrel{2 t}{\longrightarrow}(u, z) \stackrel{1}{\longrightarrow}(x, z) \stackrel{1}{\longrightarrow}(u, w)$, $(u, z) \stackrel{2 t+1}{\longrightarrow}(u, w)=(v, w)$ and $(u, z) \stackrel{2 t+2}{\longrightarrow}(u, w)=(v, w)$ for all $t \geq 0$. If $u \neq v$, $u \rightarrow v$. Since $(u, z) \stackrel{2 t}{\longrightarrow}(u, z) \stackrel{1}{\longrightarrow}(v, w)$ and $(u, z) \stackrel{2 t}{\longrightarrow}(u, z) \stackrel{1}{\longrightarrow}(u, w) \stackrel{1}{\longrightarrow}(v, w)$, $(u, z) \stackrel{2 t+1}{\longrightarrow}(v, w)$ and $(u, z) \stackrel{2 t+2}{\longrightarrow}(v, w)$ for all $t \geq 0$.

THEOREM 3. If $G$ is a connected graph and $D$ is a strongly connected digraph such that $\left|V_{G}\right| \geq 2$ and $\left|V_{D}\right| \geq 2$, then $\exp (G \otimes D)$ is $\operatorname{diam}(\mathrm{G} \otimes \mathrm{D})$ or $\operatorname{diam}(\mathrm{G} \otimes \mathrm{D})+1$. Moreover, $\exp (G \otimes D)=\operatorname{diam}(\mathrm{G} \otimes \mathrm{D})+1$ if and only if $G$ and $D$ satisfy the following:

1. $\operatorname{diam}(\mathrm{D}) \geq \operatorname{diam}(\mathrm{G})$, 
2. there is $v \in V_{D}$ such that $v \stackrel{l}{\rightarrow} v$ for all $l=1,2, \ldots, \operatorname{diam}(\mathrm{D})$,

3. either $G$ is not primitive or $G$ is primitive and $\exp (G)>\operatorname{diam}(\mathrm{D})$.

Proof. Let $\operatorname{diam}(G)=m$ and $\operatorname{diam}(D)=n$. For all $u, v \in V_{D}$, there is $u^{\prime} \in V_{D}$ such that $\left(u, u^{\prime}\right) \in A_{D}$. Since $\operatorname{diam}(D)=n, u^{\prime} \stackrel{t}{\longrightarrow} v$ for some $t \leq n$. For all $x, y \in V_{G}$, $x \stackrel{s}{\longrightarrow} y$ for some $s \leq m$. If $\alpha=\max \{m, n\}$, by Lemma $1,\left(x, u^{\prime}\right) \stackrel{l}{\longrightarrow}(y, v)$ for some $l \leq \alpha$. If $k \geq \alpha+1$, by Lemma $3,(x, u) \stackrel{k-l}{\longrightarrow}\left(x, u^{\prime}\right)$. Since $(x, u) \stackrel{k-l}{\longrightarrow}\left(x, u^{\prime}\right) \stackrel{l}{\longrightarrow}(y, v)$, $(x, u) \stackrel{k}{\longrightarrow}(y, v)$. So $\exp (G \otimes D) \leq \alpha+1=\operatorname{diam}(G \otimes D)+1$. Since $\operatorname{diam}(G \otimes D) \leq$ $\exp (G \otimes D), \exp (G \otimes D)$ is $\operatorname{diam}(G \otimes D)$ or $\operatorname{diam}(G \otimes D)+1$.

If $\exp (G \otimes D)=\alpha+1$, then there are $(x, u),(y, v) \in V_{G \otimes D}$ such that $(x, u) \stackrel{\alpha}{\rightarrow}(y, v)$ in $G \otimes D$. If $u \stackrel{l}{\longrightarrow} v$ for some $l$ with $1 \leq l \leq \alpha$, then there is $u^{\prime} \in V_{D}$ such that $\left(u, u^{\prime}\right) \in A_{D}$ and $u^{\prime} \stackrel{l-1}{\longrightarrow} v$. If $x \neq y$, then there is $x^{\prime} \in V_{G}$ such that $\left\{x, x^{\prime}\right\} \in E_{G}$ and $x^{\prime} \stackrel{s-1}{\longrightarrow} y$ where $s=\operatorname{dist}(x, y)$. If $\max \{s, l\}=p$, then $p \leq \alpha$. By Lemmas 1 and $3,\left(x^{\prime}, u^{\prime}\right) \stackrel{p-1}{\longrightarrow}(y, v)$ and $(x, u) \stackrel{\alpha-p+1}{\longrightarrow}\left(x^{\prime}, u^{\prime}\right)$. So $(x, u) \stackrel{\alpha}{\longrightarrow}(y, v)$. This is a contradiction. If $x=y$, by Lemma $3,(x, u) \stackrel{\alpha-l+1}{\longrightarrow}\left(x, u^{\prime}\right)$. Since $(x, u) \stackrel{\alpha-l+1}{\longrightarrow}$ $\left(x, u^{\prime}\right) \stackrel{l-1}{\longrightarrow}(x, v),(x, u) \stackrel{\alpha}{\longrightarrow}(x, v)=(y, v)$. This is a contradiction. So $u \stackrel{l}{\rightarrow} v$ for all $l$ such that $1 \leq l \leq \alpha$. If $u \neq v$, let $d=\operatorname{dist}(u, v)$. Then $1 \leq d \leq \alpha$ and $u \stackrel{d}{\rightarrow} v$. This is a contradiction. So $u=v$ and $G$ and $D$ satisfy condition (2).

Since $D$ is strongly connected, there is $\tilde{u} \in V_{D}$ such that $(\tilde{u}, u) \in A_{D}$. If $\operatorname{dist}(u, \tilde{u})=r \leq \alpha$, since $u \stackrel{r+1}{\longrightarrow} u, r+1 \geq \alpha+1$. Since $r \leq n \leq \alpha, r=n=\alpha$. So $n \geq m$. Thus, $G$ and $D$ satisfy condition (1). Since $(x, u) \stackrel{\alpha}{\leftrightarrow}(y, u), x \stackrel{\alpha}{\leftrightarrow} y$ in $G$. So $G$ and $D$ satisfy condition (3).

Conversely, if $G$ and $D$ satisfy conditions (1), (2) and (3), then there is $u \in V_{D}$ such that $u \stackrel{l}{\leftrightarrow} u$ for all $l$ such that $1 \leq l \leq n=\alpha$, and there are $x, y \in V_{G}$ such that $x \stackrel{\alpha}{\rightarrow} y$ in $G$. If $(x, u) \stackrel{\alpha}{\longrightarrow}(y, u)$, then $(x, u)=\left(x_{0}, u_{0}\right) \rightarrow\left(x_{1}, y_{1}\right) \rightarrow$ $\cdots \rightarrow\left(x_{\alpha}, y_{\alpha}\right)=(y, u)$ for some $x_{0}, x_{1}, \ldots, x_{\alpha} \in V_{G}$ and $u_{0}, u_{1}, \ldots, u_{\alpha} \in V_{D}$. If $u_{0}=u_{1}=\cdots=u_{\alpha}=u$, since $x=x_{0} \rightarrow x_{1} \rightarrow \cdots \rightarrow x_{\alpha}=y, x \stackrel{\alpha}{\longrightarrow} y$. This is a contradiction. If $u_{i} \neq u$ for some $i$, there are $0=i_{0}<i_{1}<\cdots<i_{s} \leq \alpha$ such that $u_{i_{p}}=u_{i_{p}+1}=\cdots=u_{i_{p+1}-1} \neq u_{i_{p+1}}$ for all $p=0,1, \ldots, s-1$ and $u_{i_{s}}=u_{i_{s}+1}=\cdots=$ $u_{\alpha}$. Since $u=u_{i_{0}} \rightarrow u_{i_{1}} \rightarrow \cdots \rightarrow u_{i_{s}} \rightarrow u_{\alpha}=u, u \stackrel{s}{\longrightarrow} u$. Since $u_{i} \neq u$ for some $i$, $1 \leq s \leq \alpha$. This is a contradiction. So $(x, u) \stackrel{\alpha}{\leftrightarrow}(y, u)$. Thus, $\exp (G \otimes D)=\alpha+1$. $\square$

COROLlary 1. If $G$ and $H$ are connected graphs, then

$$
\exp (G \otimes H)=\operatorname{diam}(\mathrm{G} \otimes \mathrm{H})
$$

except when both $G$ and $H$ are complete graphs.

Proof. If $\exp (G \otimes H)=\operatorname{diam}(G \otimes H)+1$, since $v \stackrel{2}{\longrightarrow} v$ for all $v \in V_{H}$, by Theorem $3,1 \leq \operatorname{diam}(G) \leq \operatorname{diam}(H)=1$. So $G$ and $H$ are complete graphs. $\square$ 
Note that the strong product of two complete graphs is also a complete graph, whose exponent is 2 .

\section{Corollary 2.}

$$
\exp \left(C_{n} \otimes Z_{m}\right)= \begin{cases}\left\lfloor\frac{n}{2}\right\rfloor, & \text { if } n \geq 2 m \\ m-1, & \text { if } n \text { is odd and } n \leq m \\ m, & \text { if } n \text { is even and } n \leq 2 m-2, \text { or } n \text { is odd } \\ & \text { and } m+1 \leq n \leq 2 m-1 .\end{cases}
$$

Proof. If $\exp \left(C_{n} \otimes Z_{m}\right)=\operatorname{diam}\left(C_{n} \otimes Z_{m}\right)+1, \operatorname{diam}\left(C_{n}\right)=\left\lfloor\frac{n}{2}\right\rfloor \leq \operatorname{diam}\left(Z_{m}\right)=$ $m-1$. So $n \leq 2 m-1$. Moreover, $C_{n}$ is not primitive, or $C_{n}$ is primitive and $\exp \left(C_{n}\right)=n-1>\operatorname{diam}\left(Z_{m}\right)=m-1$. So $n$ is even, or $n$ is odd and $n \geq m+1$. Thus, if $n$ is even, $n \leq 2 m-2$. And if $n$ is odd, $m+1 \leq n \leq 2 m-1$. In this case, $\exp \left(C_{n} \otimes Z_{m}\right)=(m-1)+1=m$. Otherwise, $\exp \left(C_{n} \otimes Z_{m}\right)=\operatorname{diam}\left(C_{n} \otimes Z_{m}\right)=$ $\max \left\{\left\lfloor\frac{n}{2}\right\rfloor, m-1\right\}= \begin{cases}\left\lfloor\frac{n}{2}\right\rfloor, & n \geq 2 m \\ m-1, & \text { if } n \leq 2 m-1 .\end{cases}$

Acknowledgments The authors would like to thank the anonymous referee for valuable comments and suggestions. This work was supported by the Research Institute of Natural Science of Gangneung-Wonju National University.

\section{REFERENCES}

[1] R.A. Brualdi and H.J. Ryser. Combinatorial Matrix Theory. Cambridge University Press, Cambridge, 1991.

[2] W. Imrich and S. Klavžar. Product Graphs: Structure and Recognition. John Wiley and Sons, New York, 2000.

[3] B.M. Kim, B.C. Song, and W. Hwang. Wielandt type theorem for Cartesian product of digraphs. Linear Algebra Appl., 429:841-848, 2008.

[4] B.M. Kim, B.C. Song, and W. Hwang. The exponent of Cartesian product of cycles. Appl. Math. Lett., 22:561-564, 2009.

[5] M. Kwasnik. Index of primitivity of the disjunction and the composition of two directed graphs. Math. Nachr., 134:231-235, 1987.

[6] R. Lamprey and B. Barnes. Primitivity of products of digraphs. Proceedings of the Tenth Southeastern Conference on Combinatorics, Graph Theory and Computing, Florida Atlantic Univ., Boca Raton, Fla., Congress. Numer., vol XXIII-XXIV, 637-644, 1979.

[7] M. McAndrew. On the product of directed graphs. Proc. Amer. Math. Soc., 14:600-606, 1963.

[8] C.E. Shannon. The zero error capacity of a noisy channel. IRE Trans. Inform. Theory, $2: 8-19,1956$

[9] H. Wielandt. Unzerleghare, nicht negative Matrizen. Math. Z., 52:642-645, 1950. 